\title{
Distal Hyperattenuating Vessel Sign on Computed Tomography in Acute Ischemic Stroke
}

\author{
Chang Hyo Yoon* \\ Department of Neurology, School of Medicine, Kyungpook National University, Korea
}

\begin{abstract}
The mechanisms of hyperintense vessel (HV) imaging using fluid-attenuated inversion recovery (FLAIR) have not been elucidated. However, stationary blood and slow antegrade or retrograde collateral flow distal to thromboembolic occlusion of a proximal artery has been suggested as possible explanations for HVs. Also, HVs on FLAIR sequences may be related to intra-luminal thrombi or emboli, which have also been observed using computed tomography (CT) scans indicating hyperdense middle cerebral artery (MCA) extension into the MCA branches of the Sylvian fissure (dot signs), especially in the case of erythrocyte-rich clot. But even if it looks like 'dot sign', it also can be the leptomeningeal collaterals other than the migration of thrombus from proximal occlusion. In the present report, we found that distal hyperattenuating vessels on unenhanced brain CT scans have similar shapes to HVs on FLAIR, and present at similar locations. We describe indicators of distal vessels on unenhanced brain CT, which were observed in areas of reduced edema on follow-up brain CT.
\end{abstract}

Keywords: Leptomeningeal collaterals; Distal hyperattenuating vessels; Edema

\section{Case Reports}

\section{Patient 1}

A 79-year-old right-handed woman presented at our hospital with slowly progressive memory impairment and gait disturbance. Her vascular risk factors were hypertension and diabetes mellitus. The patient was using antihypertensive and hyperglycemic medications, and was not using any antiplatelet drugs. She experienced a sudden mental stupor and left hemiplegia during hospitalization, and her National Institutes of Health Stroke Scale (NIHSS) total score was 21. Shortly after surgery, we determined that use of recombinant tissue plasminogen activator was contraindicated. A large acute right MCA infarction was indicated as an area of hyperintensity on a diffusion-weighted image (DWI), and there was hypointensity on the apparent diffusion coefficient (ADC) map. The risk of hemorrhagic transformation in a large infarct therefore prevented us from conducting mechanical thrombectomy. Follow up CT was performed at one day and four days after onset of stroke (Figure 1). The hypoattenuation observed in the right middle cerebral artery territory, but the mid-line shifting was not prominent. Fourteen days later from onset of stroke, the follow-up CT showed decreased volume of edema in the infarcted area, especially in that position, where there were serpentine or dot-shaped hyperattenuating distal vessels on $\mathrm{CT}$ imaging the same shape as HVs on FLAIR [1-3].

\section{Patient 2}

A 73-year-old man with a past medical history of atrial fibrillation, small cell lung cancer, and diabetes mellitus presented at our hospital with dyspnea for three days. He was diagnosed with pneumonia with sepsis, and antibiotics were administered. During hospitalization, his doctor recommended that he stop taking aspirin, due to thrombocytopenia. The patient presented at our department with acute dysphagia and right hemiparesis that occurred during his hospitalization. His initial NIHSS total score was 18 , and magnetic resonance imaging (MRI) indicated an infarction of the superior division of the left middle MCA, with persistent hyperintensity on DWI and hypo intensity on the ADC map. The axial view of T2-FLAIR images demonstrates the presence of HVs (i.e., focal, tubular, or serpentine hyperintensities in the subarachnoid space or extending into the brain parenchyma.)

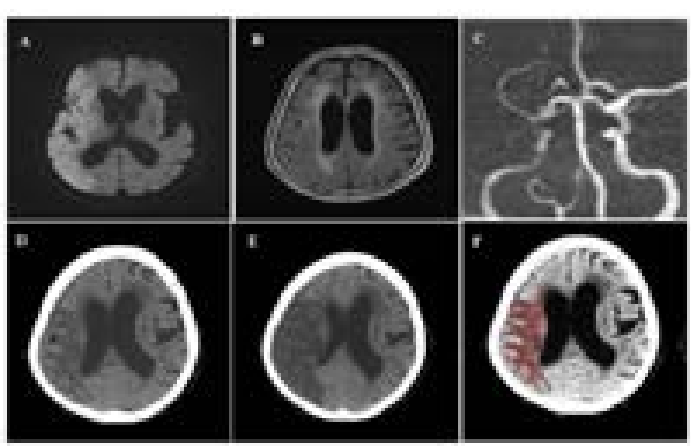

Figure 1: Patient 1. (A). Acute right middle cerebral artery (MCA) infarction is indicated as an area of hyperintensity on a diffusion-weighted image. (B). An axial fluid-attenuated inversion recovery sequence indicates distal hyperintense vessels (arrowhead). (C). Brain magnetic resonance angiography revealed complete occlusion of the right internal carotid artery. (D). Follow-up computed tomography $(C T)$ at one day after onset of stroke showed hypoattenuation in the right MCA territory. (E). Follow-up CT at 14 days after onset of stroke showed decreased volume of edema in the infarct area, especially in that position where there were serpentine-shaped hyperattenuating distal vessels. (F). A medical image processing program (ITK-SNAP 3.2) measured the hypoattenuating lesion.

distributed in the left hemisphere. Initial unenhanced brain CT scan showed the hypoattenuation observed in the superior branches of left middle cerebral artery (Figure 2). The patient was found too late, so this was not an indication of tPA or thrombectomy. The follow-up CT was performed seven days later; it showed decreased volume of the hypo attenuated lesion. And, the hyperdense vessels were prominently

*Corresponding author: Chang Hyo Yoon, Department of Neurology, School of Medicine, Kyungpook National University, Korea, Tel: 82-10-9388-4674; E-mail: golfname@gmail.com

Received November 16, 2017; Accepted November 28, 2017; Published December 04, 2017

Citation: Yoon CH (2017) Distal Hyperattenuating Vessel Sign on Computed Tomography in Acute Ischemic Stroke. J Neurol Disord 5: 368. doi:10.4172/23296895.1000368

Copyright: ( 2017 Yoon $\mathrm{CH}$. This is an open-access article distributed under the terms of the Creative Commons Attribution License, which permits unrestricted use, distribution, and reproduction in any medium, provided the original author and source are credited. 


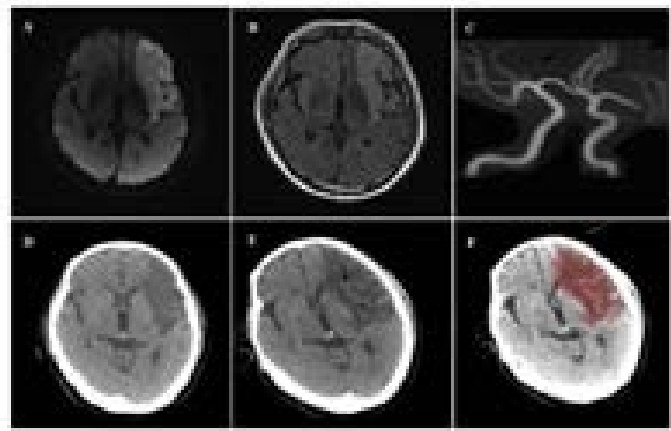

Figure 2: Patient 2. (A). Acute infarction of the superior division of the left middle cerebral artery (MCA) is indicated as an area of hyperintensity on a diffusionweighted image. (B). An axial fluid-attenuated inversion recovery sequence indicates distal hyperintense vessels (HVs) in the Sylvian fissure (arrowhead) (C). Brain magnetic resonance angiography revealed occlusion of the superio branches of the left MCA and indicated a remaining thrombus (arrow) on the distal M1 segment of the left MCA. (D). Initial brain computed tomography (CT) showed hypoattenuation in the territory of the superior branch of the left middle cerebral artery. (E). Follow-up CT at seven days after onset of stroke showed decreased volume of the hypoattenuated lesion, and the hyperattenuating distal vessels were prominently observed in the normalized hypoattenuating lesion (arrowhead). (F). A medical image processing program (ITK-SNAP 3.2) measured the hypoattenuating lesion.

observed in the normalized hypoattenuating lesion on this follow-up CT.

\section{Discussion}

A large middle cerebral artery infarction is commonly associated with variable degrees of brain edema and a high mortality rate. Malignant brain edema following ischemic stroke leads to neurological deterioration caused by tissue shifts compressing the midline structures, eventually leading to a variable degree of herni ation. Previously, Van der Eecken et al studied that the infarcted area in most patients was smaller than actual distribution supplied by the occluded artery and presumed that the retrograde slow flows actions to minimize the infarcted area. These retrograde slow blood flows of engorged pial vasculature to the acute ischemic lesion are called by leptomeningeal collaterals (LMCs), but the pathophysiological mechanisms are not fully understood. HVs indicated on FLAIR images have been widely investigated as possible imaging markers for LMCs. However, the clinical significance of HVs on FLAIR, specifically the prognostic value to reduce edema during acute ischemic stroke, has not been elucidated.

We previously hypothesized that vessel signs or 'dot' lesions on unenhanced brain CT would be related to intra-luminal thrombi or emboli $[3,4]$. In addition, MRI data have been reported in isolated cortical venous thrombosis, which was recognized as marked homogeneous, well-delineated signals that were tubular and serpentine. However, If the distal hyperattenuating vessels are veins, edema would increase because the flow would be reduced, or a thrombus would form in the vein, thereby blocking blood drainage. Also, if distal vessels on brain CT are arterial thromboembolisms, the edema should be worse. If the hemorrhagic transformation is the distal vessel on the brain CT, they should also be observed as hyperattenuating rather than normalized hypoattenuating lesions upon follow-up CT.

We have interested in how LMCs showed on unenhanced brain CT and wheather this vessel sign has any role. The major observations in our patient cases are (a) distal hyperattenuating vessels were observed on unenhanced brain CT, and had similar shapes to HVs on FLAIR in acute ischemic stroke, and (b) the normalized hypoattenuated regions indicated by follow-up CT were the same sites of distal hyperattenuating vessel observation. The limitations of our study include, firstly, that the vessels are not easily visible using brain $\mathrm{CT}$ during the period required for determining thrombolysis or craniectomy. Rather, they are more clearly observed with brain CT performed several days later. However, if distal hyperattenuating vessels on brain CT images show shapes similar to HVs on FLAIR, then HVs on FLAIR may help to determine thrombolysis or craniectomy. Secondly, CT is suitable for detecting brain edema, but only the use of DWI and ADC maps on MRI enable earlier and clearer differentiation of cytotoxic and vasogenic edema. Finally, we did not assess spontaneous recanalization in our patients, despite that recanalization affects the pattern of edema [5].

\section{Conclusion}

The distal hyperattenuating vessels observed in the present study contributed to establishing an effective penumbra zone until recanalization, and were also associated with reduced edema. Therefore, predictive models considering both the status of the occlusion site and the status of the leptomeningeal collaterals, which are observed as HVs using FLAIR or as distal hyperattenuating vessels using brain $\mathrm{CT}$, could improve the accuracy of predicting malignant brain edema. Therefore, this predictive model will help to determine the timing of decompressive craniectomy and further treatments to reduce increased intracranial pressure.

\section{References}

1. Kamran S, Bates V, Bakshi R, Wright $P$, Kinkel W, et al. (2000) Significance of hyperintense vessels on flair mri in acute stroke. Neurology 55: 265-269.

2. Oppenheim C, Logak M, Dormont D, Lehericy S, Manai R, et al. (2000) Diagnosis of acute ischaemic stroke with fluid-attenuated inversion recovery and diffusion-weighted sequences. Neuroradiology 42: 602-607.

3. Barber PA, Demchuk AM, Hudon ME, Pexman JH, Hill MD, et al. (2001) Hyperdense sylvian fissure mca "dot" sign: A ct marker of acute ischemia. Stroke 32: 84-88.

4. Liebeskind DS, Sanossian N, Yong WH, Starkman S, Tsang MP, et al. (2001) $\mathrm{CT}$ and mri early vessel signs reflect clot composition in acute stroke. Stroke 42: $1237-1243$.

5. Simard JM, Kent TA, Chen M, Tarasov KV, Gerzanich V, et al. (2007) Brain oedema in focal ischaemia: Molecular pathophysiology and theoretical implications. Lancet Neurol 6: 258-268. 